\title{
The muon detector prototype AMD for the determination of the muon content in UHECRs
}

\author{
Christine Peters ${ }^{* 1}$, Thomas Bretz ${ }^{1}$, Thomas Hebbeker ${ }^{1}$, Markus Lauscher ${ }^{1}$, \\ Rebecca Meißner ${ }^{1}$, Lukas Middendorf ${ }^{1}$, Tim Niggemann ${ }^{1}$, Johannes Schumacher ${ }^{1}$ \\ ${ }^{1}$ III. Phys. Inst. A, RWTH Aachen University, Aachen, Germany \\ E-mail: peters@physik.rwth-aachen.de
}

\begin{abstract}
Precise measurements of the muon content of extensive air showers are essential for the identification of the chemical composition of ultra-high-energy cosmic rays. Therefore, a new scintillator detector prototype is proposed, the Aachen Muon Detector (AMD). It can complement existing ground arrays composed of, e.g., water-Cherenkov detector stations. The detector consists of 64 scintillator tiles read out by semiconductor photo sensors, silicon photomultipliers (SiPM), which are located in a steel housing which could be placed beneath existing detector stations. SiPMs promise a photon detection efficiency which outperforms current photomultiplier tubes. In combination with their compact package, low cost per light sensor and a moderate bias voltage $(<100$ volts) a modular and robust design can be achieved. The current status of the AMD prototype is presented, including the first characterisation measurements of the scintillator tiles and the first promising simulation studies. We use a detailed detector simulation based on Geant 4 to determine the efficiency of the AMD detector to reconstruct the simulated muon number in air showers.
\end{abstract}

The 34th International Cosmic Ray Conference,

30 July- 6 August, 2015

The Hague, The Netherlands

\footnotetext{
* Speaker.
} 


\section{Introduction}

Understanding the mass composition of ultra-high-energy cosmic rays ${ }^{1}$ is fundamental to reveal their production and propagation mechanisms. At Earth, they are indirectly characterised by measuring the extensive air showers that they initiate in the atmosphere. The mass composition of cosmic rays can then be derived from air shower observables measured by different detection mechanisms on a statistical basis.

For the determination of the mass composition, a detailed understanding of the air shower development is indispensable [1]. Simulations require knowledge of the interaction of all air shower particles. In air showers, some interactions of hadronic particles take places at energies which are not accessible in accelerator experiments. As the interaction cross-sections are still not known precisely, a large spread is found in the predictions of air shower simulation models. The systematic uncertainty on these models can be reduced by improved measurements of hadronic interactions at this energy scale. Therefore, a study of the muonic shower component, which is directly related to these interactions, is needed. The number of muons $N_{\mu}$ is thereby a crucial parameter. This observable increases with a higher mass number $A$ of the primary particle and is complementary to other mass-sensitive observables. If certain observables are combined, hadronic interaction models can be validated and the mass composition of cosmic rays can be studied in detail.

Water-Cherenkov detector stations [2] as used at the Pierre Auger Observatory [3] measure the combined energy deposition of charged particles on the ground, i.e. the electromagnetic and muonic component. For a discrimination between these components on an event-by-event basis, an additional detector is needed. A muon detector prototype is presented, namely the Aachen Muon Detector (AMD), which could be located beneath the water-Cherenkov detector stations.

\section{Silicon Photomultipliers}

Silicon photomultipliers (SiPMs) are semiconductor photo detectors which consist of an array of up to thousands of small, independent Geiger-mode avalanche photodiodes (G-APDs) connected in parallel. Each G-APD, referred to as a cell, is capable of single photon detection. The number of individual cells sets the limit of the dynamic range of an SiPM. As SiPMs are operated in Geigermode, the gain of SiPMs is typically in the order of $10^{6}$ [4]. The gain depends linearly on the over-voltage $V_{\mathrm{ov}}$. The over-voltage is defined as the excess of the applied bias voltage $V_{\text {bias }}$ above the breakdown voltage $V_{\text {break }}$, which is typical around $70 \mathrm{~V}$ for Hamamatsu SiPMs. An increase of the over-voltage implies an improved photon detection efficiency (PDE), i.e. the probability of detecting a single photon, but also leads to an increase of noise.

This dark noise of SiPMs can be categorised in three components:

Thermal noise due to thermal excitation, optical crosstalk caused by photons emitted during deexcitation in a cell which in turn may trigger neighboring cells, and afterpulsing created by defects in the silicon lattice where electrons or holes of the avalanche may be trapped and released afterwards, triggering a new avalanche. Optical crosstalk and afterpulsing are summarised as correlated noise. A reduction of thermal and correlated noise has already been achieved for current devices

\footnotetext{
${ }^{1}$ Cosmic rays with an energy above $10^{18} \mathrm{eV}$.
} 


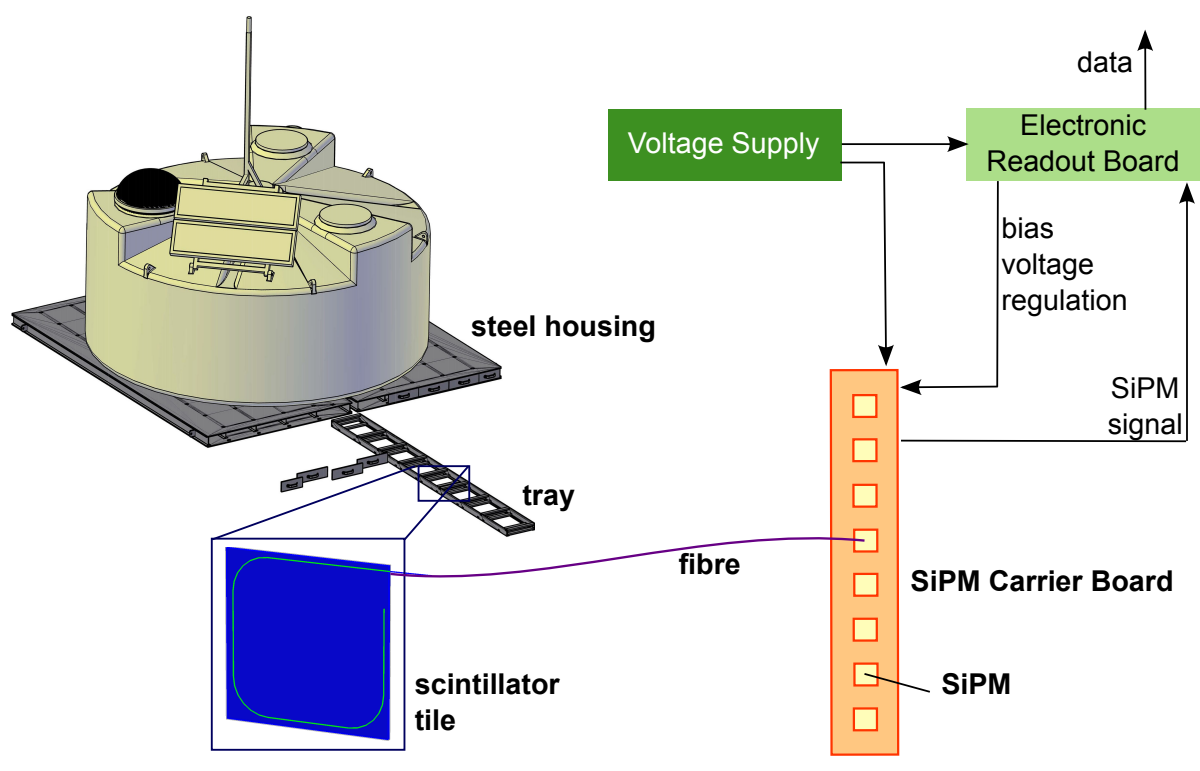

Figure 1: Sketch of the AMD prototype detector principle.

compared to models of a few years ago and an even larger suppression is expected for future devices [4].

\section{Baseline Design of AMD}

The Aachen Muon Detector is a scintillator-based detector using silicon photomultipliers (SiPMs) as photo sensors. A complete station consists of 64 scintillating tiles (plastic scintillator EJ-212 from Eljen Technology [5]), each of dimensions $30 \times 30 \times 0.5 \mathrm{~cm}^{3}$, resulting in a total active scintillator area of $5.76 \mathrm{~m}^{2}$. The tiles are located with a gap of $7.5 \mathrm{~cm}$ next to each other. Eight of them are lined up on a $35 \mathrm{~cm}$ wide tray. A complete station consists of eight trays and each tray can be individually inserted or removed. The trays are located in a steel support structure. It is made of two pieces of approximately $4 \times 1.8 \times 0.1 \mathrm{~m}^{3}$. The complete steel structure could be installed beneath a water-Cherenkov detector station as used by the Pierre Auger Observatory. The tank serves as shielding for electrons and positrons for the AMD detector, as well as a complementary detector to distinguish between the electromagnetic and muonic component of an air shower (c.f. figure 1 and 2).

When a charged particle traverses the active scintillator area, photons are produced along the track. The emitted photons are collected in a bent wavelength shifting fibre (WLS, BCF-92 from Saint Gobain [6]) with $1 \mathrm{~mm}$ diameter. This fibre is placed inside a sigma-shaped groove milled into the scintillator tile and fixed and coupled by optical glue (cf. figure 3). One end of the fibre is mirrored, the other end is coupled to a $1 \mathrm{~mm}$ diameter clear optical fibre (ESKA ${ }^{\circledR}$ fibre from Mitsubishi [7]). The light is transported by the optical fibre along the tray to the photo sensor. As each optical fibre in a tray has an optimal length from tile to sensor and all sensors of a tray are located at one end, the optical fibres have a length ranging between 0.3 and $2.9 \mathrm{~m}$. The location of all SiPMs on one carrier board at one end allows an easier monitoring of the temperature, voltage control and read-out of the SiPMs. 


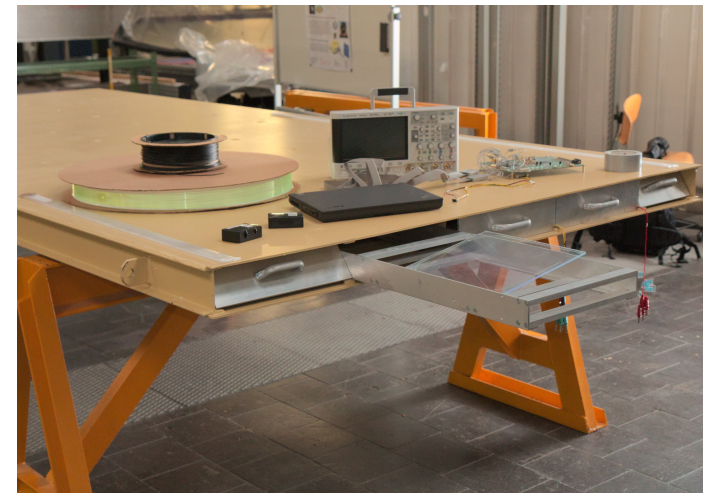

Figure 2: The AMD detector prototype. Shown is half of the steel housing with 32 scintillating tiles in 4 trays.

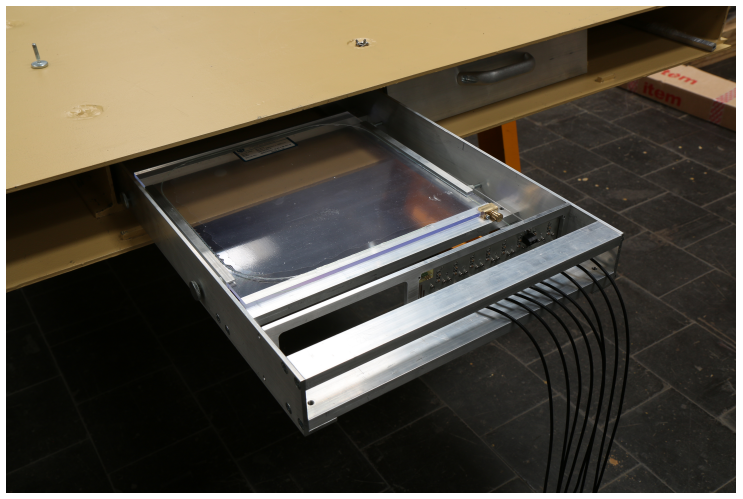

Figure 3: Photo of a scintillator tile with optical coupling between WLS and clear fibre. The WLS fibre is glued into the scintillator groove.

The SiPMs used in the AMD prototype are of type Hamamatsu S12571-050P [8]. Those devices have a photosensitive area of $1 \times 1 \mathrm{~mm}^{2}$ with a cell pitch of $50 \mu \mathrm{m}$, and thus with 400 cells. The photon detection efficiency is $\simeq 35.6 \%$ at a wavelength of $\lambda=454 \mathrm{~nm}$ and an overvoltage of $V_{\mathrm{ov}}=2.6 \mathrm{~V}$ at room temperature [8].

SiPMs have a relatively high noise rate, which depends exponentially on the ambient temperature. At room temperature, the SiPM type installed in AMD has a noise rate of $100 \mathrm{kHzmm}^{-2}$. The bias voltage to operate the SiPMs is about $67 \mathrm{~V}$. As each SiPM needs a slightly different bias voltage, the voltage is supplied by a dedicated electronics (readout) board for each SiPM individually. To achieve a constant gain, the SiPM supply voltage is automatically adjusted with changing ambient temperature. The readout board is based on the EASIROC ${ }^{2}$ chip, a 32 channel analogue front-end ASIC for SiPMs developed by Omega Micro and optimised for low power consumption [9]. The chip features one trigger discriminator output per channel, which will be used to count muon hits. The trigger threshold is in common for all channels by an integrated 10-bit DAC (Digital to Analog Converter). In a second chain, it also allows the digitisation of one analogue value per channel per event after a tunable slow shaper and a track and hold circuit, to measure the muon number in case of saturation due to a high hit rate. The digitisation is performed by an external ADC (Analog to Digital Converter). An individual adjustment of the bias voltage in small steps is possible as the board also integrates a $4.5 \mathrm{~V}$ range 8-bit DAC per channel to adjust the individual ground level per SiPM. Each board also has an FPGA (Field Programmable Gate Array) for communication with the EASIROC chip, temporary data storage and possible communication with the water-Cherenkov detector station. For the complete detector station two readout boards are necessary.

\section{First Test Measurements}

During completion of the assembly of the AMD prototype, first test measurements were performed.

\footnotetext{
${ }^{2}$ Extended Analogue SI-pm ReadOut Chip
} 


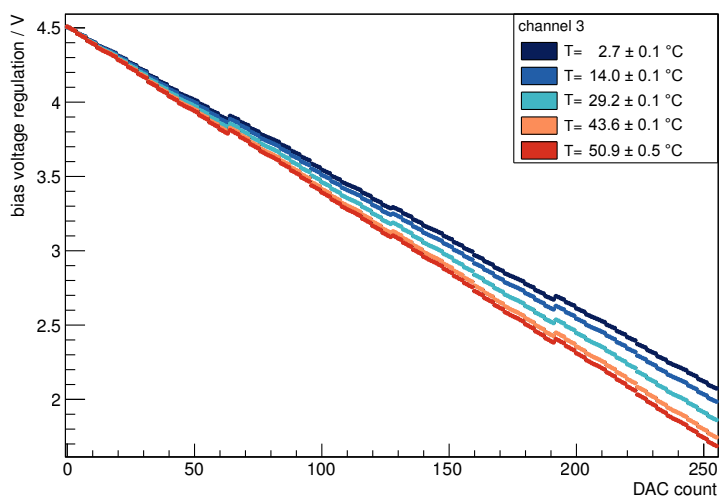

Figure 4: Temperature dependence of the 8-bit DAC for the setting of the fine voltage adjustment of the SiPMs. The sharp steps in the voltage output occur when single bits are switched on or off. One channel of the readout board is exemplarily shown.

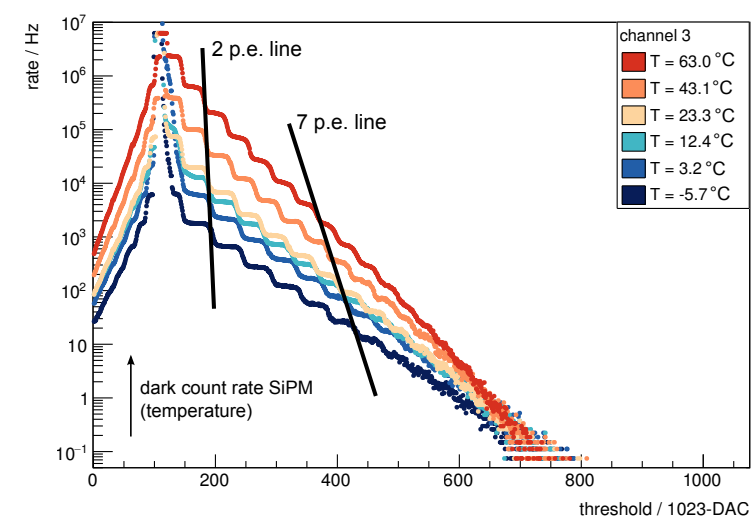

Figure 5: Rate of an SiPM as a function of DAC counts for different temperatures. The absolute trigger rate increases with temperature as the dark noise rate of the SiPM increases. The gain of the SiPM is kept constant. Nevertheless, the set DAC count results in different thresholds for different temperatures shown by the two diagonal lines indicating a temperature dependence arising in the EASIROC chip.

\subsection{Characterisation of the Temperature Dependance}

The electronic readout board, as well as certain properties of SiPMs depend on temperature. For a detailed understanding of the AMD prototype response, different temperature dependencies have been investigated:

1. The SiPM noise increases with temperature.

2. The SiPM gain is corrected for changes in temperature to achieve a stable gain.

3. The output of the 8-bit DAC installed at the readout board for the fine voltage adjustment is also temperature dependent. Its linear temperature dependence is shown in figure 4. For higher temperatures the adjusted voltage for a selected DAC count decreases. The sharp steps in the voltage output occur when single bits are switched on or off.

4. The 10-bit DAC installed for the threshold setting at the EASIROC chip depends on temperature and the conversion factor from selected DAC count to the achieved threshold in photon equivalents (p.e.) has to be determined. In figure 5, the trigger rate of an SiPM as a function of DAC counts for different temperatures is shown. The absolute trigger rate of the SiPM increases with temperature as the dark noise rate of the SiPM is increased. At the same time, the preset DAC count for a specific trigger threshold in p.e. is shifted to higher DAC counts for higher temperatures, although the gain of the SiPM is kept constant. This shift is indicated by the two diagonal lines in figure 5 and depicts the temperature dependence of the 10-bit DAC as a function of the preset DAC count. For a larger threshold, the variation of the DAC counts increases with temperature.

The temperature dependencies of the different components are well characterised and a stable operation of the SiPM read-out is achieved. 


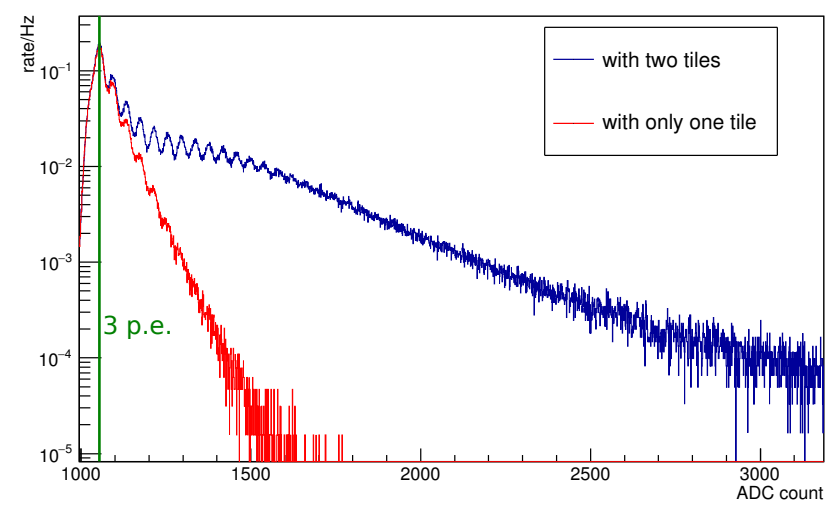

Figure 6: Integrated charge spectrum of coincidence measurements of two scintillating tiles readout by SiPMs (blue). Shown is the number of triggered events as a function of the the number of ADC counts in comparison with a pure dark noise measurements (red).

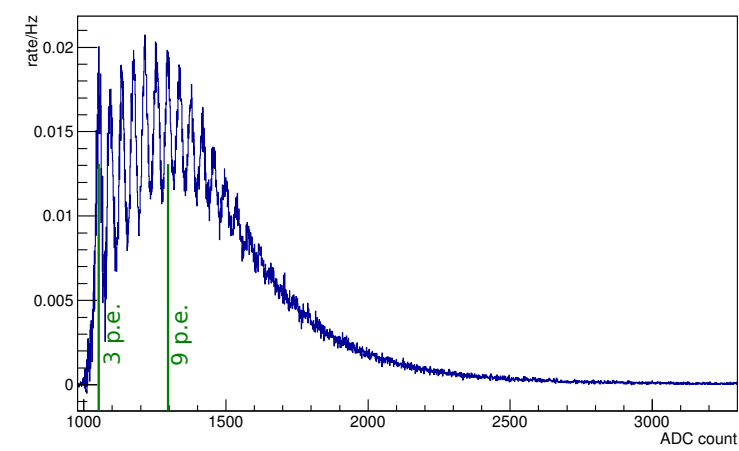

Figure 7: Integrated charge spectrum of coincidence measurements of two scintillating tiles readout by SiPMs. Shown is the number of triggered events as a function of the ADC counts whereby the SiPM dark noise background has been removed. The peaks indicating the 3 p.e. and 9 p.e. signal are depicted in green.

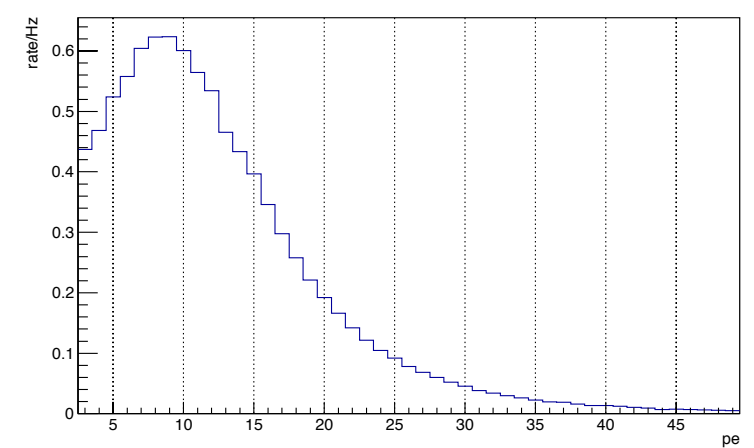

Figure 8: Spectrum of coincidence measurements of two scintillating tiles readout by SiPMs. Shown is the binned number of triggered events as a function of the muon signal in photon equivalents. The SiPM dark noise background has been removed.

\subsection{Light Yield of the Scintillator Tile}

In order to reveal a clear signal above noise, the minimum ionisation particle (MIP) peak of a tile should be clearly above the dark noise level. For two tiles, the first MIP response was measured using atmospheric muons. The first prototypes of two scintillating tiles wrapped in Tyvek are measured in coincidence. The tiles are placed directly above each other and the emitted light of each tile is guided on one SiPM each. A trigger threshold of 2.5 p.e. is set. After a trigger occurs, the signal integrated by the slow shaper is read out by the ADC. In figure 6, the number of coincident triggered events (in blue) is shown as a function of the number of ADC counts. Also a pure dark noise measurement (in red) without a tile on the selected channel is shown for comparison. For small ADC counts the dark noise of the SiPM dominates, for large ADC counts, synonymous with high light output in the tiles, the signal of atmospheric muons arises. The muon signal is clearly distinguishable from the SiPM dark noise background. After removing the SiPM 
dark noise background, the MIP peak becomes visible. In figure 7 the signal spectrum is shown as a function of ADC counts. In the integrated charged spectrum each peak corresponds to a certain number of photon equivalents. Therefore, the number of triggered events can be binned and shown as a function of the muon signal in p.e. (figure 8). There are still improvements under investigation concerning the wrapping of the tiles, the optical coupling between the fibres and the clear optical waveguide and the photo sensor, as well as the electronic readout.

\section{End-to-end Simulations}

To evaluate the baseline design of the AMD detector, a dedicated Geant4 [10] ray-tracingsimulation has been developed. In this simulation, the Geant4 package G4SiPM [11] is implemented which allows a detailed simulation of the SiPM characteristics and response based on a phenomenological model. To address the detailed response of the detector to extensive air showers, a large dataset of simulated cosmic ray air showers is necessary. These showers were produced using CORSIKA [12] (version 7.4005) and the hadronic interaction model EPOS LHC [13]. For this work, air showers with an energy of $10^{19.5} \mathrm{eV}$ at a fixed incident angle of $0^{\circ}$ were simulated. Since the AMD detector is located beneath a water-Cherenkov detector station for shielding, the Auger Offline Framework [14] has been used to extract air shower particles which reach the AMD detector. This particle distribution is injected in the AMD detector simulation and the response is analysed. For a perfectly constructed AMD (perfect wrapping, optical coupling etc.), the simulation studies yield a most probable value (MPV) for the detection of single muons in one tile of approx. 40 p.e. resulting in a high-efficient detection of single muons. As a first performance study, the ability of AMD to reconstruct muons by counting is shown in figure 9 .

For 1000 vertical CORSIKA proton showers, the signal produced by muons has been analysed with a simple trigger criterion of a discriminator threshold of 10.5 p.e.. The number of muons passing the trigger criterion is determined. Using a lateral-shower-profile-fit, the reconstructed number of muons $N_{\text {Trigger }}^{\mathrm{AMD}}$ is calculated from the muon triggers per AMD station and is divided by the generated number of muons in the air shower from CORSIKA $N_{\mu}^{\text {CORSIKA }}$. This ratio is shown in figure 9. A shift from unity is observed as for detector stations with a high muon number the counting method becomes inefficient because the SiPM signal is almost always above the trigger threshold for the duration of the shower incidence. At this occasion, the AMD read-out would have to be switched to the integration chain. The relative resolution is $18 \%$ which is already quite promising.

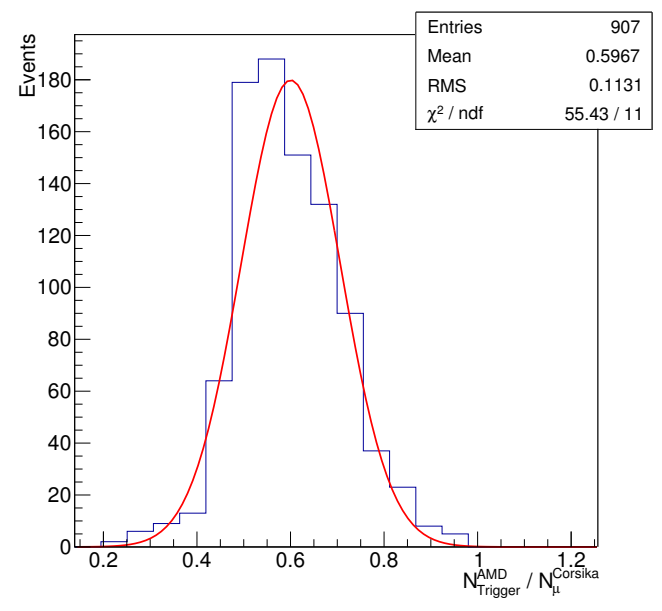

Figure 9: Ratio of triggered number of muons from AMD and the generated muon number from CORSIKA in the same effective area. 1000 vertical proton showers with an energy of $10^{19.5} \mathrm{eV}$ and an incident angle of $\theta=0^{\circ}$ have been analysed. A shift from unity is observed for the current setup as for events with a high muon rate counting becomes inefficient. The relative resolution is $18 \%$. 


\section{Summary \& Outlook}

The Aachen Muon Detector prototype is a scintillator-based detector with SiPM read-out. Its designed setup consists of 64 scintillating tiles with a total active area of $5.76 \mathrm{~m}^{2}$. The emitted light in each tile is collected by a WLS fibres and guided by clear optical waveguides onto one SiPM per tile. The temperature dependencies in the readout electronics have been characterised and first measurements of the light yield of the scintillating tiles have been studied. The MIP peak due to atmospheric muons can be clearly distinguished from dark noise of the photo sensors. CORSIKA air shower and detailed Geant 4 detector simulations have been performed, and successfully demonstrated the ability of AMD to detect muons in air showers. Further simulation studies are ongoing to investigate the improvement obtained by AMD for the determination of the mass composition of cosmic rays or for other possible applications as large area muon detector, veto and counter. The prototype will be fully assembled and operational later this year.

Acknowledgment: This work is funded by the German Federal Ministery of Education and Research BMBF, the Helmholtz Alliance for Astroparticle Physics, the seed fund of RWTH Aachen University and the Evangelisches Studienwerk e.V. Villigst. Additionally, the authors thank the mechanical and electronics workshops in Aachen.

\section{References}

[1] Pierre Auger Collaboration, A. Aab et al., Muons in air showers at the Pierre Auger Observatory: Mean number in highly inclined events, Phys. Rev. D. 91 (2015) 032003, [arXiv:1408.1421].

[2] Pierre Auger Collaboration, I. Allekotte et al., The Surface Detector System of the Pierre Auger Observatory, Nucl. Instrum. Meth. A 586 (2008) 409 - 420, [arXiv:0712.2832].

[3] Pierre Auger Collaboration, A. Aab et al., The Pierre Auger Cosmic Ray Observatory, accepted for publication in Nucl. Instrum. Meth. A (2015), [arXiv:1502.01323].

[4] D. Renker and E. Lorenz, Advances in solid state photon detectors, JINST 4 (2009) P04004.

[5] Eljen Technology, EJ-212 Plastic Scintillator Datasheet (June 2015).

[6] Saint Gobain Crystals, Scintillating Optical Fibers Brochure, (June 2015).

[7] Edmund Optics, Mitsubishi - ESKA ${ }^{\circledR}$ Fiber Strands Datasheet (June 2015).

[8] Hamamatsu, MPPC series S12571 datasheet (June 2015).

[9] S. Callier et al., EASIROC, an easy \& versatile ReadOut Device for SiPM, in Proceeding of the TIPP 2011, Physics Procedia 37 (2012) 1569 - 1576.

[10] S. Agostinelli et al., Geant4-a Simulation Toolkit, Nucl. Instrum. Meth. A 506 (2003) 250-303.

[11] T. Niggemann et al., G4SiPM: A novel silicon photomultiplier simulation package for Geant4, Nucl. Instrum. Meth. A 787 (2015) 344-347.

[12] D. Heck et al., CORSIKA: a Monte Carlo code to simulate extensive air showers, Forschungszentrum Karlsruhe Report 6019 (1998).

[13] T. Pierog, K. Werner, EPOS Model and Ultra High Energy Cosmic Rays (2009), [arXiv:0905.1198].

[14] S. Argirò et al., The Offline Software Framework of the Pierre Auger Observatory, Nucl. Instrum. Meth. A 580 (2007) 1485-1496, [arXiv:0707.1652]. 